\title{
Circular features in the Trans-Mexican Volcanic Belt
}

\author{
Francisco Anguita , Surendra P. Verma , Alvaro Márquez, Manuel Vasconcelos-F. , \\ Ivan López , Alejandro Laurrieta
}

\begin{abstract}
One hundred and ninety-one circular or elliptical features have been located on Landsat imagery of the Trans-Mexican Volcanic Belt (TMVB). The origin of most of these features is unknown. Nine have been recognized as collapse calderas (clearly visible on Landsat imagery) and studied in detail, while an equally small number have been tentatively identified as such but not thoroughly investigated. On the basis of the identification of at least five of the nine calderas through their detection on Landsat images, it is proposed that the present inventory is a reliable base to extend the census of the TMVB confirmed calderas, now clearly too small for a population of roughly 8000 volcanic centers, many of which emitted large volumes of felsic pyroclastic products.
\end{abstract}

Keywords: Mexico; Calderas; Remote sensing; Trans-Mexican Volcanic Belt

\section{Introduction}

The Trans-Mexican Volcanic Belt (TMVB) is a volcanic province $20-150 \mathrm{~km}$ wide and about $1000 \mathrm{~km}$ long, stretching in a roughly east-west direction from Puerto Vallarta to Veracruz at the Pacific Ocean and Gulf of Mexico coasts, respectively (Demant, 1978; Robin, 1982; Verma, 1987, and references cited therein). In spite of the large extension of the province covered by felsic pyroclastic products, only nine of the approximately 8000 volcanic centers identified (Robin, 1982) in the TMVB have been classified as collapse calderas. Several authors (Nelson and Carmichael, 1984; Negendank et al., 1985) have located other volcano-tectonic features within the TMVB, but no systematic attempt has ever been made to identify calderas across the entire volcanic belt.

Such work is considered of paramount importance by the present authors, because many geothermal energy and volcanic hazards are associated with calderas. In 1991, two of us (F.A. and S.P.V.) searched Landsat images for semicircular features in the central part of the TMVB. Fieldwork led to the identification of Mazahua caldera (Fig. 1; Anguita et al., 1991), and served as a test case for the present work.

\section{The inventory}

The entire TMVB is covered by 13 Landsat MSS series images, numbers 28, 29, 34, 35, 41, 42, 49, 50, $58,59,67,75$, and 76 in the collection available from 


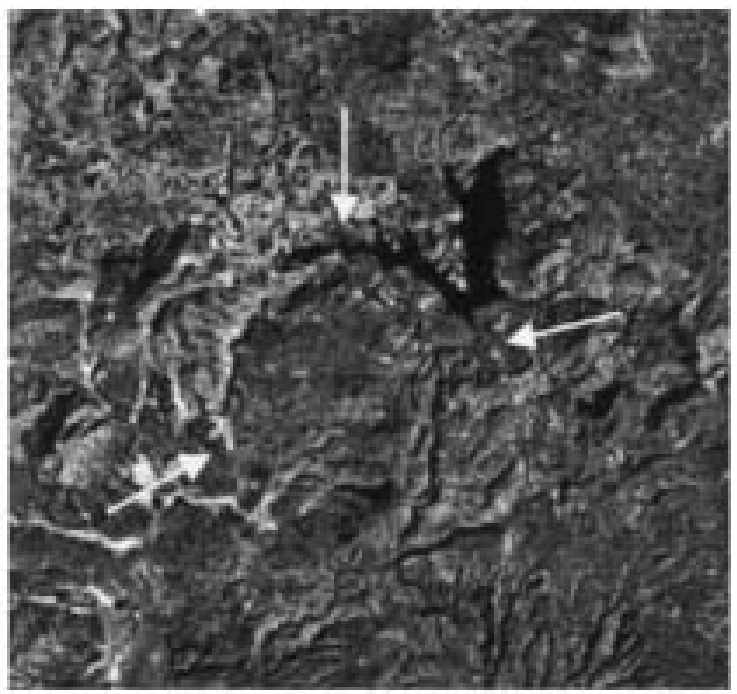

Fig. 1. The Mazahua caldera (approximately $8 \mathrm{~km}$ diameter), idenified first on the basis of this Landsat image (image 41, coordinates $19^{\circ} 37 \mathrm{~N}, 100^{\circ} 00^{\prime} \mathrm{W}$; feature \#105 in Table 1; reliability: Fair) and then confirmed from field work. North is up. Arrows highlight the caldera rim.

Institut• Nacional de Estadistica, Geøgrafía e Informática, (NEGI, the •fficial mapping institution in Mexicø). The bulk of our work was the identification of circular and elliptical features on those images (Fig. 2). These features were then located on 'spacimaps' (georeferenced color Landsat image møsaics) published by INEGI and by SAHOP (the Secretaría de Asentamientos Humanos y Obras Públicas), and assigned a number, geographical data and descriptive features, such as mørphøløy, diameter, and apparent relations to tectonic lineaments. We then assigned each feature a reliability index. The label 'Good' indicates a clearly distinguishable feature on both Landsat images and spaciomaps. 'Fair' indicates a feature seen

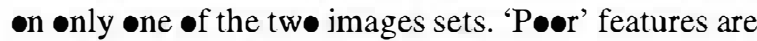
not clearly expressed on either image set. Our inventory, includes 191 features, listed in order of decreasing longitude. We classify the features as circular ( $65 \%)$, semicircular $(\sim 15 \%)$, elliptical $(\sim 19 \%)$, and semielliptical $(\sim 1 \%)$ subsets. We attribute the 'half' features to faulting.

Our inventory (Table 1) may have overlooked søme caldera-like features, and søme features identified by us may not be calderas. Field work is needed.
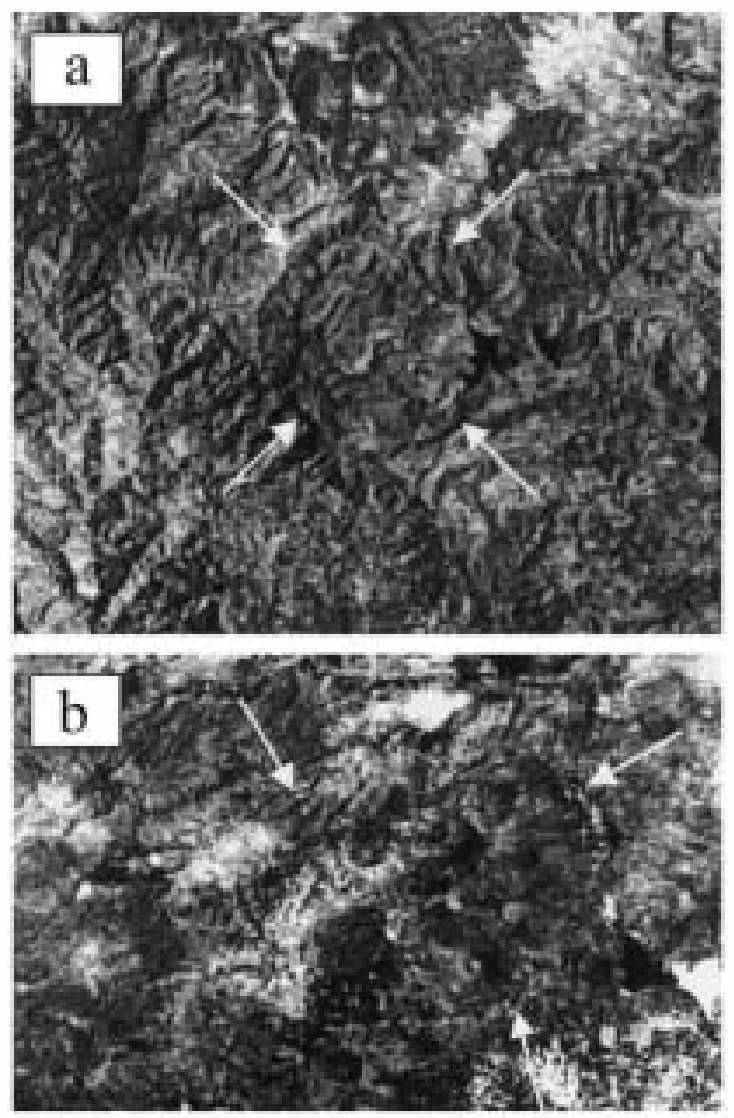

Fig. 2. Examples of circular (a, Landsat image 58, coordinates $19^{\bullet} 18.3^{\prime} \mathrm{N}, 11^{\bullet} 32.8^{\prime} \mathrm{W}$, about $7 \mathrm{~km}$ diameter, feature $\# 62$ in Table 1; reliability: Good) and elliptical (b, Landsat image 49, coordinates $20^{\bullet} 1^{\prime} \mathrm{N}, 11^{\bullet} 36.5^{\prime} \mathrm{W}$; about $2 \times 3 \mathrm{~km}$ diameter; feature \#159 in Table 1; reliability: Poor) features. North is up. Arrows point the borders of the features.

\section{The circular features in the tectonic framework of the TMVB}

The tectonics of the TMVB has been studied by Nelsøn and Carmichael (1984); Lug• et al. (1985); Luhr et al. (1985); Negendank et al. (1985); Nelsøn and Sánchez-Rubi• (1986); Pasquarè et al. (1987); Jøhnsøn and Harrison (1990); Allan et al. (1991); Ferrari et al. (1991); Wallawender and Hanan (1992); Moore et al. (1994); Suter et al. (1995); Ramírez-Herrera (1996); and Campos-Enríquez et al. (1999). As sh॰wn in Fig. 3, a number of the faults detected by these authors transect many of the features -f our census. 


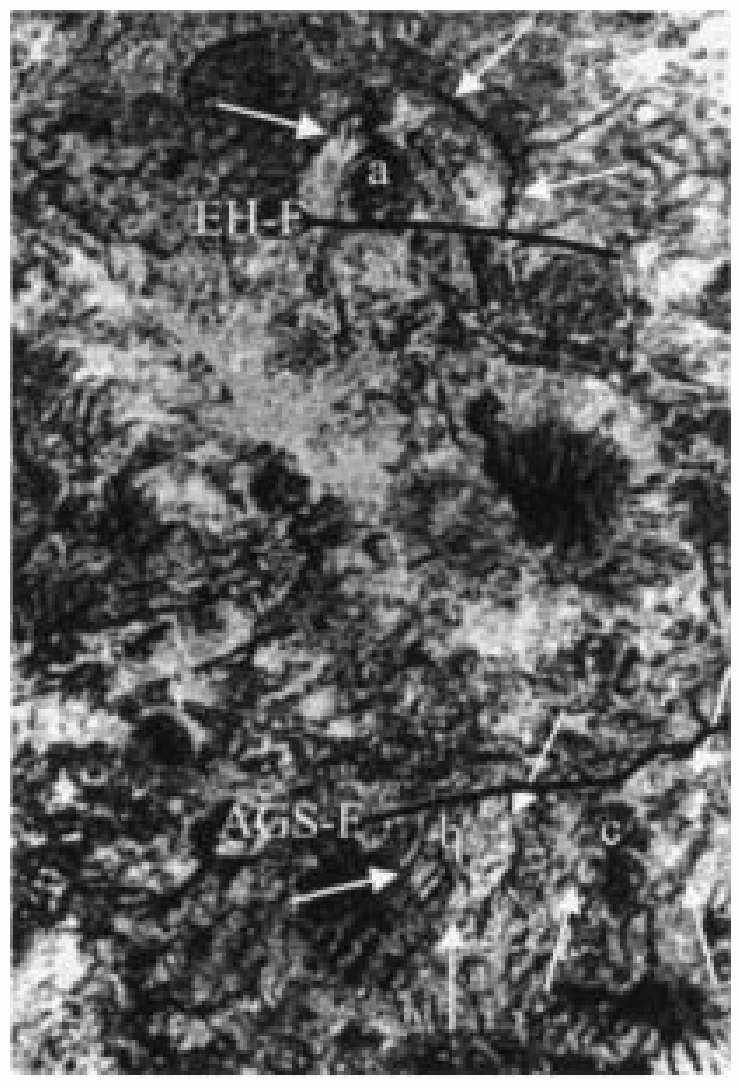

Fig. 3. Examples of features apparently connected with satellite lineations. a, the Amealco caldera (about $10 \mathrm{~km}$ diameter, Landsat image 49, coordinates $20^{\circ} 08^{\prime} \mathrm{N}, 100^{\circ} 09^{\prime} \mathrm{W}$; feature \#100 in Table 1 ; reliability: Good, cut by the Epitcaio Huerta fault identified EH-F); and b, c, two other semicircular features cut by a fault (identified AGS-F) belonging to the Acambay graben system. North is up. Arrows point the borders of the features.
Fig. 4 is a tectonic sketch map of the TMVB, to which the circular features have been added. From this superposition it becomes clear that the highest concentration of circular features $(15 \%)$ occurs at the intersection of two important tectonic systems of central Mexico: the east-west Chapala-Tula tectonic lineament $\left(7-7^{\prime}\right.$ in Fig. 4) and a $\mathrm{N} 160^{\circ} \mathrm{W}$ lineament, the Querétaro-Taxco feature system $\left(6-6^{\prime}\right.$ in Fig. 4). Probably it is not a coincidence that three of the nine recognized calderas in the TVMB (Los Azufres, Amealco, and Mazahua) are located on this tectonic crossing. Other concentrations of circular features occur at equally significant tectonic intersections: for instance, La Primavera caldera is located (together with eight other circular features) on the crossing of the main arm of the TMVB with the Colima graben. Twenty of the features form likewise a belt at about $102^{\circ} \mathrm{W}$ longitude, where the Pénjamo north-south graben crosses the TMVB (4-4' in Fig. 4). On the contrary, there is a clear gap where the Tzitzio anticline (Ferrari et al., 1991) crosses the Belt, thus suggesting a possible association of at least some of the features with extensional stress fields. The absence of features in the vicinity of the Lake Cuitzeo could be related to the compression caused on this area by the Michoacan block.

\section{Discussion}

One possible origin of the circular and elliptical features is the collapse of a volcanic construct. Other possibilities include circular intrusions, tectonic

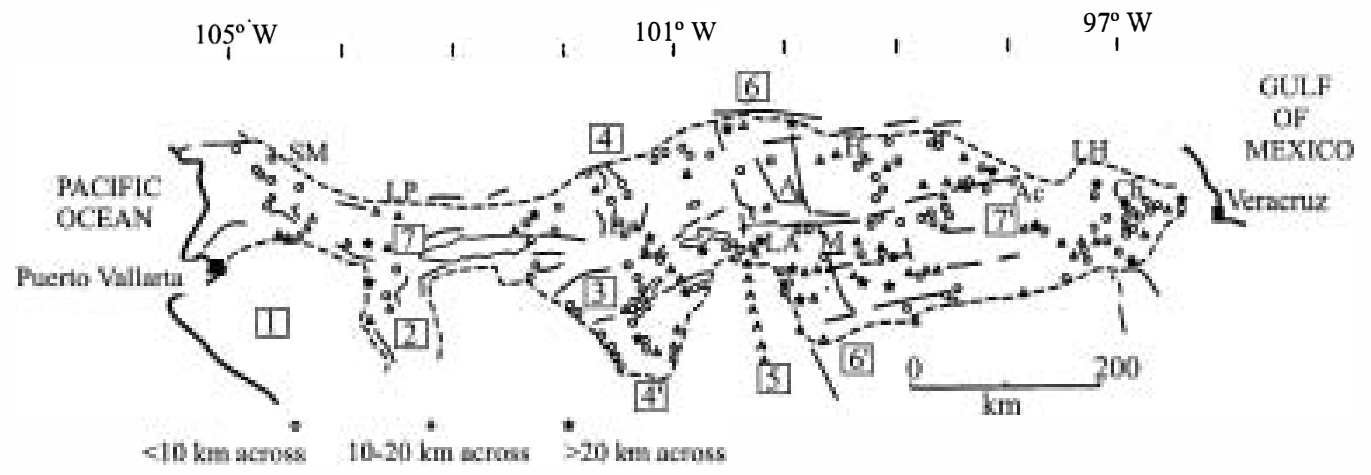

Fig. 4. Tectonic sketch map of the TMVB, with the locations of detected features shown with symbols: circles (58\%) for features less than $10 \mathrm{~km}$ across, triangles (29\%) for features between 10 and $20 \mathrm{~km}$ across, and stars (13\%) for features larger than $20 \mathrm{~km}$ across. Verified calderas are: SM, Santa María del Oro; LP, La Primavera; LA, Los Azufres; A, Amealco; M, Mazahua; H, Huichapan; Ac, Acoculco; LH, Los Humeros; Ch, Chiconquiaco. Major tectonic elements are: 1, Jalisco block; 2, Colima graben; 3, Michoacan block; 4-4', Pénjamo graben; 5 , Tzitzio anticline; $6-6^{\prime}$, Querétaro-Taxco fracture zone; $7-7^{\prime}$, Chapala-Tula fracture zone. 


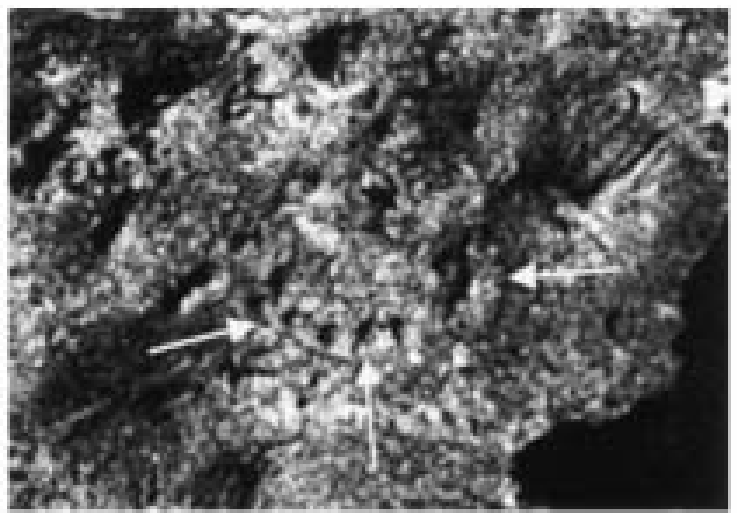

Fig. 5. This elliptical feature (about $5 \times 9 \mathrm{~km}$ diameter, Landsat image 58 , coordinates $19^{\bullet} 38.8^{\prime} \mathrm{N}, 11^{\bullet} 44.2^{\prime} \mathrm{W}$; feature $\# 52$ in Table 1; reliability: Fair) is partially circle by lava domes, possible evidence for its relation with a once active magma chamber. North is up. Arrows point the border of the feature. subsidence, and the influence of the Sierra Madre Occidental old tectonovelcanic structures on the younger TMVB constructs. Althøugh our satellite image reconnaissance aløne does n॰t define the origin -f the features, some evidence favors the caldera cøllapse hypothesis. Such is the presence of møn॰genetic emission centers (domes?) circling søme of the features possibly representing ring-fractures (Fig. 5), or of volcanic (resurgent?) constructs centered on some others (Fig. 6).

We contend that our inventory is a simple but useful tool to help design field campaigns for løating calderas. This procedure has been successfully used in several cases (Løs Humerøs by Pérez-Reynosø, 1978; Santa Maria del Orø by Nelsøn and Carmichael, 1984; Chiconquiac by Negendank et al., 1985; Løs Azufres by Pradal and Røbin, 1985; Mazahua by Anguita et

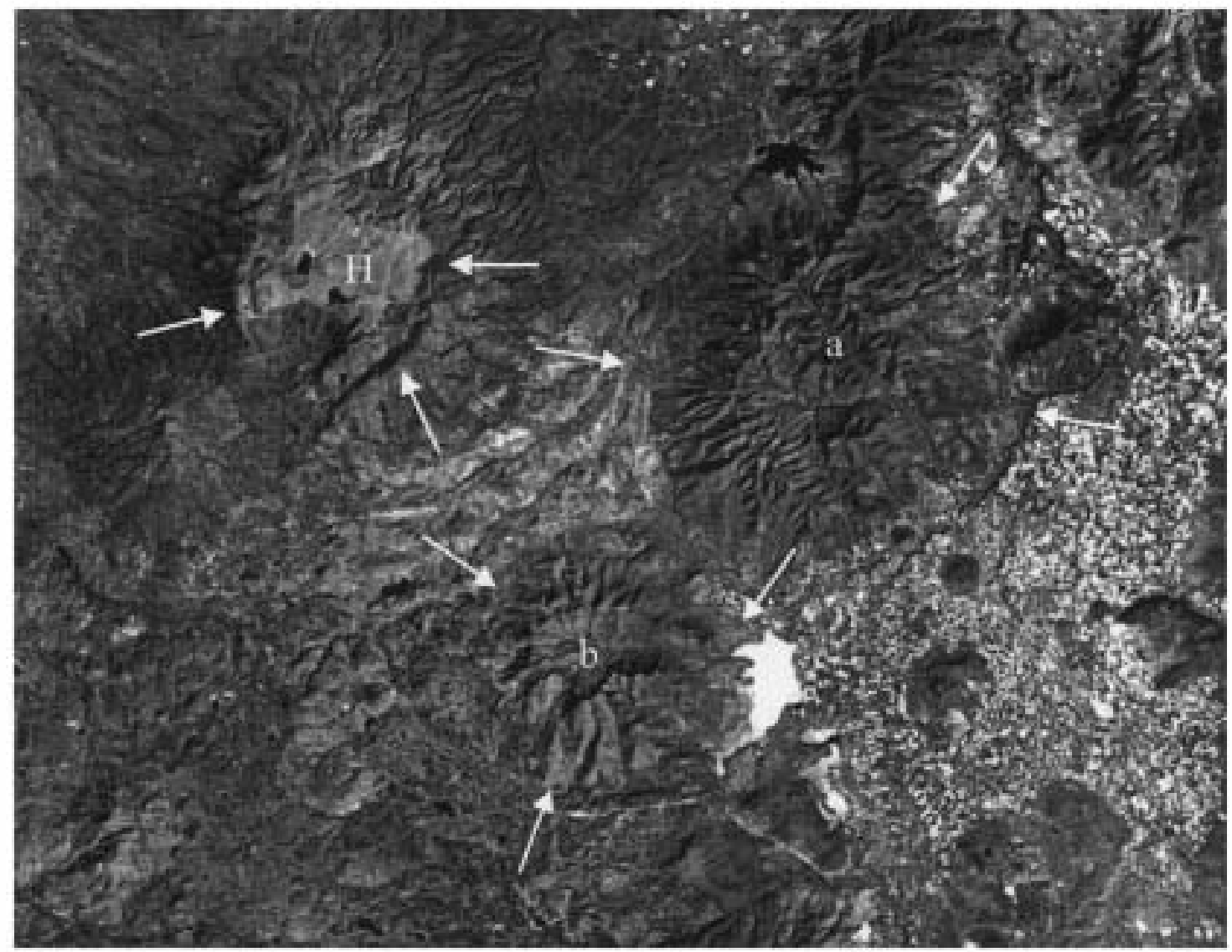

Fig. 6. The centers of the features a, b, (Landsat image 41 , respective coordinates $20^{\circ} 11^{\prime} \mathrm{N}, 9^{\circ} 26^{\prime} \mathrm{W}$; feature \#122 in Table 1 ; reliability: Good, and $20^{\circ} 17^{\prime} \mathrm{N}, 9^{\circ} 13^{\prime} \mathrm{W}$; feature $\# 129$ in Table 1; reliability: Good) appear occupied by volcanic constructs, possible resurgent edifices. H is the Huichapan caldera (about $8 \times 10 \mathrm{~km}$ diameter). North is up. Arrows point the borders of the features. 
Table 1

Inventory of circular features in the Trans-Mexican Volcanic Belt

\begin{tabular}{|c|c|c|c|c|c|c|c|c|c|c|}
\hline \multirow[t]{2}{*}{ Feature } & \multirow{2}{*}{$\begin{array}{l}\text { Landsat } \\
\text { image }\end{array}$} & \multicolumn{2}{|l|}{ Coordinates } & \multirow{2}{*}{$\begin{array}{l}\text { Diameter } \\
(\mathrm{km})\end{array}$} & \multirow[t]{2}{*}{ Morphology } & \multirow[t]{2}{*}{ Lineaments } & \multirow{2}{*}{$\begin{array}{l}\text { Political } \\
\text { State }\end{array}$} & \multirow[t]{2}{*}{ Closest village } & \multirow{2}{*}{$\begin{array}{l}\text { INEGI } \\
\text { map no. }\end{array}$} & \multirow{2}{*}{$\begin{array}{l}\text { Feature } \\
\text { reliability }^{b}\end{array}$} \\
\hline & & Long. (W) & Lat. (N) & & & & & & & \\
\hline 1 & 75 & $104^{\circ} 51^{\prime}$ & $21^{\bullet} 31^{\prime}$ & 5 & Circular & NW-SE & Nayarit & Bellavista & F13-D21 & Good \\
\hline 2 & 75 & $104^{\circ} 43^{\prime}$ & $21^{\bullet} 19^{\prime}$ & $3 \times 5$ & Elliptical & - & Nayarit & San Pedro Lagunillas & F13-D31 & Good \\
\hline 3 & 75 & $104^{\bullet} 42^{\prime}$ & $21^{\bullet} 16^{\prime}$ & 5 & Circular & - & Nayarit & San Pedro Lagunillas & F13-D31 & Fair \\
\hline 4 & 75 & $104^{\circ} 42^{\prime}$ & $21^{\circ} 00^{\prime}$ & 9 & Circular & E-W; NE-SW & Nayarit & Talpa de Allende & F13-241, D61 & Good \\
\hline 5 & 76 & $104^{\circ} 40^{\prime}$ & $20^{\circ} 43^{\prime}$ & 16 & Semicircular & $E-W$ & Jalisco & San Sebastián & F13-D51 & Good \\
\hline 6 & 75 & $104^{\circ} 40^{\prime}$ & $21^{\bullet} \cdot 2^{\prime}$ & 12 & Semicircular & $E-W$ & Nayarit & Lizeta & F13-241, D61 & Fair \\
\hline 7 & 76 & $104^{\circ} 40^{\prime}$ & $20^{\circ} 41^{\prime}$ & $20 \times 25$ & El]iptical & NW-SE & Jalisco & Zacatongo Natividad & F13-D61, D62 & Good \\
\hline 8 & 75 & $104^{\bullet} 39^{\prime}$ & $21^{\bullet} 13^{\prime}$ & 4 & Circular & - & Nayarit & San Pedro Lagunillas & F13-D42 & Good \\
\hline 9 & 75 & $104^{\bullet} 34^{\prime}$ & $21^{\bullet} 22^{\prime}$ & 7 & Circular & NW-SE & Nayarit & Santa Ma. del Oro & F13-D42 & Fair \\
\hline 10 & 76 & $104^{\bullet} 31^{\prime}$ & $20^{\circ} 42^{\prime}$ & 15 & Circular & NW-SE & Jalisco & Zacatongo & F13-D42 & Good \\
\hline 11 & 76 & $104^{\bullet} 26^{\prime}$ & $20^{\circ} 45^{\prime}$ & 10 & Semicircular & NW-SE & Jalisco & Zacatongo Natividad & F13-D42 & Good \\
\hline 12 & 75 & $104^{\bullet} 22^{\prime}$ & $21^{\bullet} 4^{\prime}$ & $5 \times 6$ & Elliptical & NW-SE & Nayarit & Ixtlán del Río & F13-D42, $43, \mathbf{D} 52, \mathbf{D} 53$ & Good \\
\hline 13 & 67 & $104^{\bullet} 21^{\prime}$ & $20^{\circ} 46^{\prime}$ & 12 & Circular & NW-SE & Nayarit & Amatlán de Cañas & F13-D52, D53 & Fair \\
\hline 14 & 67 & $104^{\circ} \cdot 6^{\prime}$ & $20^{\bullet} 31^{\prime}$ & 12 & Circular & NW-SE & Jalisco & Ameca & F13-D63, D64, D73, D74 & Fair \\
\hline 15 & 67 & $104^{\circ} 00^{\prime}$ & $19^{\circ} 51^{\prime}$ & 13 & Circular & $\mathrm{N}-\mathrm{S}$ & Jalisco & Tapalpa & F13-D83, D84, E13-B13, B14 & Fair \\
\hline 16 & 67 & $103^{\circ} 58^{\prime}$ & $20^{\circ} 07^{\prime}$ & 20 & Circular & NE-SW; N-S & Jalisco & Tecolotlán & F13-D84 & Fair \\
\hline 17 & 67 & $103^{\circ} 52^{\prime}$ & $20^{\circ} 27^{\prime}$ & 22 & Circular & NW-SE; N-S & Jalisco & El Cabezón & F13-D71 & Fair \\
\hline 18 & 67 & $103^{\circ} 48^{\prime}$ & $20^{\circ} 1^{\prime}$ & 8 & Circular & NW-SE & Jalisco & Juanacatlán & F13-D84 & Poor \\
\hline 19 & 67 & $103^{\circ} 46^{\prime}$ & $19^{\circ} 53^{\prime}$ & $8 \times 10$ & Elliptical & - & Jalisco & Tapalpa & F13-B14 & Poor \\
\hline 20 & 67 & $103^{\circ} 44^{\prime}$ & $20^{\circ} 57^{\prime}$ & 14 & Circular & - & Jalisco & Tala (Presa de la Vega) & F13-D64 & Fair \\
\hline 21 & 67 & $103^{\circ} 44^{\prime}$ & $20^{\bullet} 23^{\prime}$ & 12 & Circular & WNW-ESE & Jalisco & Villa Corona & F13-D74 & Fair \\
\hline 22 & 67 & $103^{\circ} 40^{\prime}$ & $20^{\bullet} 13^{\prime}$ & 8 & Semicircular & NW-SE; E-W & Jalisco & Barranca de Sta. Clara & F13-D84, D85, D74, D75 & Fair \\
\hline 23 & 67 & $103^{\bullet} 33^{\prime}$ & $20^{\bullet} 38^{\prime}$ & 18 & Circular & - & Jalisco & Guadalajara & F13-D54, D55 & Fair \\
\hline 24 & 58 & $102^{\circ} 50^{\prime}$ & $20^{\bullet} 22^{\prime}$ & 10 & Circular & - & Jalisco & Ocotlán & F13-D77 & Good \\
\hline 25 & 58 & $102^{\circ} 43^{\prime}$ & $19^{\circ} 58^{\prime}$ & 6 & Circular & NW-SE; E-W & Michoacán & Los Olivos & E13-B17 & Good \\
\hline 26 & 58 & $102^{\circ} 42^{\prime}$ & $20^{\circ} \cdot 2^{\prime}$ & 4 & Circular & $E-W$ & Michoacán & Julquilpan de Juárez & F13-D87 & Good \\
\hline 27 & 58 & $102^{\bullet} 38^{\prime}$ & $20^{\bullet} 16^{\prime}$ & 7 & Circular & - & Jalisco & Jamay & F13-D78 & Fair \\
\hline 28 & 58 & $102^{\bullet} 36^{\prime}$ & $20^{\circ} 26^{\prime}$ & $17 \times 25$ & Elliptical & $E-W$ & Jalisco & Portezuelo & F13-D78 & Good \\
\hline 29 & 58 & $102^{\bullet} 25^{\prime}$ & $20^{\bullet} 17^{\prime}$ & 5 & Semicircular & NW-SE & Michoacán & Yurecuaro & F13-D78 & Good \\
\hline 30 & 58 & $102^{\bullet} 26^{\prime}$ & $19^{\bullet} 37^{\prime}$ & 5 & Circular & NE-SW; E-W & Michoacán & Los Reyes de Salgado & F13-B28, B29 & Good \\
\hline 31 & 58 & $102^{\bullet} 22^{\prime}$ & $19^{\bullet} 35^{\prime}$ & 7 & Circular & NE-SW; E-W & Michoacán & Los Reyes de Salgado & F13-B28, B29 & Fair \\
\hline 32 & 58 & $102^{\bullet} 21^{\prime}$ & $20^{\bullet} 12^{\prime}$ & 8 & Circular & ENE-WSW & Jalisco & El Aguacaliente & F13-D88 & Good \\
\hline 33 & 59 & $102^{\bullet} 14^{\prime}$ & $19^{\circ} 25^{\prime}$ & 9 & Circular & WNW-ESE & Michoacán & Nuevo Parangaricutiro & E13-B39 & Fair \\
\hline 34 & 59 & $102^{\circ} \cdot 6^{\prime}$ & $19^{\bullet} 14^{\prime}$ & 6 & Circular & NW-SE; E-W & Michoacán & Gabriel Zamora & E13-B49 & Fair \\
\hline 35 & 59 & $102^{\bullet} 05^{\prime}$ & $19^{\bullet} 10^{\prime}$ & 6 & Circular & NW-SE & Michoacán & Gabriel Zamora & E13-B49 & Good \\
\hline 36 & 59 & $102^{\circ} 4^{\prime}$ & $19^{\circ} 07^{\prime}$ & 6 & Circular & NW-SE & Michoacán & El Gueco & E13-B49 & Fair \\
\hline 37 & 58 & $102^{\circ} 1^{\prime}$ & $20^{\circ} 40^{\prime}$ & 7 & Circular & N-S; NE-SW & Jalisco & Jose fina de Allende & F13-D69, F14-C61 & Good \\
\hline 38 & 50 & $102^{\circ} 00^{\prime}$ & $19^{\bullet} 17^{\prime}$ & 42 & Circular & NE-SW; NW-SE & Michoacán & Taretan & E14-A31, A41, E13-B49, B39 & Fair \\
\hline 39 & 58 & $101^{\bullet} 59^{\prime}$ & $20^{\circ} 44^{\prime}$ & $15 \times 19$ & Elliptical & NE-SW; ENE-WSW & Jalisco & Jesús Maria & F13-D69, F14-C61 & Fair \\
\hline
\end{tabular}


Table 1 (continued)

\begin{tabular}{|c|c|c|c|c|c|c|c|c|c|c|}
\hline \multirow[t]{2}{*}{ Feature } & \multirow{2}{*}{$\begin{array}{l}\text { Landsat } \\
\text { image }\end{array}$} & \multicolumn{2}{|l|}{ Coordinates } & \multirow{2}{*}{$\begin{array}{l}\text { Diameter } \\
(\mathrm{km})\end{array}$} & \multirow[t]{2}{*}{ Morphology } & \multirow[t]{2}{*}{ Lineaments } & \multirow{2}{*}{$\begin{array}{l}\text { Political } \\
\text { State }\end{array}$} & \multirow[t]{2}{*}{ Closest village } & \multirow{2}{*}{$\begin{array}{l}\text { INEGI } \\
\text { map no. }\end{array}$} & \multirow{2}{*}{$\begin{array}{l}\text { Feature } \\
\text { reliability }^{b}\end{array}$} \\
\hline & & Long. (W) & Lat. (N) & & & & & & & \\
\hline 40 & 58 & $101^{\bullet} 58^{\prime}$ & $20^{\bullet} 24^{\prime}$ & $6 \times 8$ & Elliptical & ENE-WSW & Guanajuato & Pénjamo & F14-C71 & Good \\
\hline 41 & 58 & $101^{\bullet} 55^{\prime}$ & $19^{\bullet} 31^{\prime}$ & 14 & Circular & $E-W$ & Michoacán & Ziracuaretiro & E14-A21, A31 & Fajr \\
\hline 42 & 50 & $101^{\bullet} 51^{\prime}$ & $19^{\bullet} 12^{\prime}$ & 8 & Circular & NE-SW & Michoacán & Ario Rosales & E14-A41 & Fajr \\
\hline 43 & 50 & $101^{\bullet} 5 \mathbf{⿰}^{\prime}$ & $19^{\bullet} 24^{\prime}$ & $6 \times 8$ & Elliptical & NE-SW & Michoacán & Ziracuaretro & E14-A31 & Fajr \\
\hline 44 & 58 & $101^{\circ} 49^{\prime}$ & $19^{\circ} 28^{\prime}$ & $4 \times 5$ & Elliptical & NE-SW & Michoacán & Ziracuarewro & E14-A31 & Fajr \\
\hline 45 & 50 & $101^{\circ} 49^{\prime}$ & $19^{\circ} 27^{\prime}$ & $6 \times 10$ & Elliptical & NE-SW & Michoacán & Ziracuaretiro & E14-A31 & Fajr \\
\hline 46 & 58 & $101^{\circ} 47^{\prime}$ & $20^{\circ} 11^{\prime}$ & 3 & Circular & - & Michoaán & Angamacutiro & F14-C81 & Fair \\
\hline 47 & 58 & $101^{\bullet} 47^{\prime}$ & $20^{\circ} 99^{\prime}$ & 4 & Circular & - & Michoacán & Angamacutiro & F14-C81 & Poor \\
\hline 48 & 58 & $101^{\bullet} 47^{\prime}$ & $19^{\circ} 51^{\prime}$ & 5 & Elliptical & NE-SW & Michoacán & Zacapú & E14-A11 & Fajr \\
\hline 49 & 58 & $101^{\circ} 47^{\prime}$ & $19^{\bullet} 33^{\prime}$ & 6 & Circular & NE-SW & Michoacán & Tingambato & E14-A21 & Fajr \\
\hline 50 & 59 & $101^{\bullet} 47^{\prime}$ & $19^{\circ} 07^{\prime}$ & 6 & Circular & - & Michoacán & Ario Rosales & E14-A41 & Fajr \\
\hline 51 & 50 & $101^{\circ} 45^{\prime}$ & $19^{\bullet} 34^{\prime}$ & 6 & Circular & NE-SW & Michoacán & Erongaricuaro & E14-A21 & Fajr \\
\hline 52 & 58 & $101^{\circ} 44^{\prime}$ & $19^{\circ} 39^{\prime}$ & $5 \times 9$ & Elliptical & $E-W$ & Michoacán & Erongaricuaro & E14-A21 & Fajr \\
\hline 53 & 58 & $101^{\bullet} 43^{\prime}$ & $20^{\bullet} 34^{\prime}$ & 6 & Circular & NE-SW & Guanajuato & Pénjamo & F14-C61 & Fajr \\
\hline 54 & 58 & $101^{\circ} 43^{\prime}$ & $20^{\circ} 10^{\prime}$ & 7 & Circular & - & Michoacán & Angamacutiro & F14-C81 & Fajr \\
\hline 55 & 49 & $101^{\circ} 43^{\prime}$ & $19^{\circ} 42^{\prime}$ & 7 & Semicircular & $E-W$ & Michoacán & Naranja de Tapia & E14-A21 & Fajr \\
\hline 56 & 58 & $101^{\circ} 41^{\prime}$ & $20^{\circ} 22^{\prime}$ & 7 & Semicircular & WNW-ESE & Guanajuato & Pénjamo & F14-C71 & Poor \\
\hline 57 & 58 & $101^{\bullet} 41^{\prime}$ & $19^{\bullet} 30^{\prime}$ & $7 \times 5$ & Elliptical & $E-W$ & Michoacán & Pátzcuaro & E14-A31, A21 & Poor \\
\hline 58 & 59 & $101^{\bullet} 39^{\prime}$ & $19^{\circ} 4^{\prime}$ & 12 & Circular & N-S; NE-SW & Michoacán & La Huacana & $\mathrm{E} 14-\mathrm{A} 42$ & Good \\
\hline 59 & 49 & $101^{\bullet} 37^{\prime}$ & $20^{\circ} \cdot 1^{\prime}$ & $20 \times 3$ & Elliptical & $E-W$ & Michoacán & Villa Morelos & F14-C82 & Poor \\
\hline 60 & 58 & $101^{\bullet} 37^{\prime}$ & $19^{\circ} 54^{\prime}$ & 10 & Semicircular & $E-W$ & Michoacán & Villa de Jiménez & E14-A12 & Fajr \\
\hline 61 & 50 & $101^{\bullet} 34^{\prime}$ & $19^{\circ} 6^{\prime}$ & 15 & Circular & NE-SW; N-S & Michoacán & Puruarán & E14-A42 & Good \\
\hline 62 & 58 & $101^{\bullet} 33^{\prime}$ & $19^{\circ} 18^{\prime}$ & 7 & Circular & NE-SW & Michoacán & Coeneo & E14-A32 & Good \\
\hline 63 & 50 & $101^{\bullet} 33^{\prime}$ & $19^{\circ} 4^{\prime}$ & 7 & Circular & NE-SW; N-S & Michoacán & Puruarán & $\mathrm{E} 14-\mathrm{A} 42$ & Good \\
\hline 64 & 50 & $101^{\bullet} 3 \mathbf{e}^{\prime}$ & $19^{\bullet} 14^{\prime}$ & 15 & Semicircular & NE-SW & Michoacán & Tacámbaro & E14-A42 & Fajr \\
\hline 65 & 49 & $101^{\bullet} 28^{\prime}$ & $19^{\circ} 46^{\prime} 15$ & 15 & Circular & NE-SW & Michoacán & Quiroga & E14-A12 & Poor \\
\hline 66 & 49 & $101^{\bullet} 22^{\prime}$ & $20^{\circ} 42^{\prime}$ & 5 & Circular & NE-SW; E-W & Guanajuato & Irapuato & F14-C62 & Fajr \\
\hline 67 & 49 & $101^{\bullet} 22^{\prime}$ & $19^{\circ} \cdot 7^{\prime}$ & 15 & Circular & NE-SW & Michoacán & Capula & E14-A42 & Poor \\
\hline 68 & 50 & $101^{\bullet} 21^{\prime}$ & $19^{\circ} \cdot 6^{\prime}$ & 30 & Circular & NE-SW; N-S & Michoacán & Morelia & E14-A42 & Fajr \\
\hline 69 & 49 & $101^{\bullet} 20^{\prime}$ & $20^{\circ} 45^{\prime}$ & 3 & Circular & NE-SW;E-W & Guanajuato & Irapuato & F14-C52, C53, C62, C63 & Fajr \\
\hline 70 & 49 & $101^{\bullet} 17^{\prime}$ & $19^{\bullet} 33^{\prime}$ & 4 & Circular & $\begin{array}{l}\text { ENE-WSW; NNW- } \\
\text { SSE }\end{array}$ & Michoacán & Acuitrio & E14-A23 & Fajr \\
\hline 71 & 49 & $101^{\bullet} 11^{\prime}$ & $19^{\bullet} 37^{\prime}$ & 7 & Semicircular & ENE-WSW & Michoacán & Morelia & E14-A23 & Poor \\
\hline 72 & 49 & $101^{\bullet} 10^{\prime}$ & $20^{\circ} 45^{\prime}$ & $3 \times 5$ & Elliptical & $E-W$ & Guanajuato & La Ordña & F14-C53, C63 & Fajr \\
\hline 73 & 49 & $101^{\bullet} \cdot 8^{\prime}$ & $19^{\circ} 55^{\prime}$ & $27 \times 35$ & Elliptical & $E-W$ & Michoacán & Cuitzeo & E14-A13 & Fajr \\
\hline 74 & 49 & $100^{\circ} 55^{\prime}$ & $2 \bullet^{\circ} 41^{\prime}$ & 10 & Semicircular & NW-SE & Guanajuato & Salamanca & F14-C64 & Fajr \\
\hline 75 & 49 & $100^{\circ} 54^{\prime}$ & $20^{\circ} 48^{\prime}$ & 5 & Circular & NE-SW & Guanajuato & La Ordeña & F14-C54 & Fajr \\
\hline 76 & 49 & $100^{\circ} 53^{\prime}$ & $19^{\circ} 51^{\prime}$ & 5 & Circular & ENE-WSW & Michoacán & Queréndaro & E14-A14 & Poor \\
\hline 77 & 49 & $100^{\bullet} 5 \mathbf{0}^{\prime}$ & $19^{\circ} 45^{\prime}$ & 8 & Semicircular & ENE-WSW; NW-SE & Michoacán & Queréndaro & E14-A14 & Fair \\
\hline
\end{tabular}


Table 1 (continued)

\begin{tabular}{|c|c|c|c|c|c|c|c|c|c|c|}
\hline \multirow[t]{2}{*}{ Feature } & \multirow{2}{*}{$\begin{array}{l}\text { Landsat } \\
\text { image }\end{array}$} & \multicolumn{2}{|l|}{ Coordinates } & \multirow{2}{*}{$\begin{array}{l}\text { Diameter } \\
(\mathrm{km})\end{array}$} & \multirow[t]{2}{*}{ Morphology } & \multirow[t]{2}{*}{ Lineaments } & \multirow{2}{*}{$\begin{array}{l}\text { Political } \\
\text { State }\end{array}$} & \multirow[t]{2}{*}{ Closest village } & \multirow{2}{*}{$\begin{array}{l}\text { INEGI } \\
\text { map no. }\end{array}$} & \multirow{2}{*}{$\begin{array}{l}\text { Feature } \\
\text { reliability }\end{array}$} \\
\hline & & Long. (W) & Lat. (N) & & & & & & & \\
\hline 78 & 49 & $100^{\circ} 49^{\prime}$ & $19^{\circ} 51^{\prime}$ & 6 & Circular & ENE-WSW & Michoacán & Zinapécuaro & E14-A14 & Poor \\
\hline 79 & 49 & $100^{\circ} 48^{\prime}$ & $20^{\circ} 43^{\prime}$ & 5 & Circular & - & Guanajuato & Commonfort & F14-C64 & Fair \\
\hline 8 & 49 & $100^{\circ} 47^{\prime}$ & $19^{\circ} 46^{\prime}$ & 7 & Circular & ENE-WSW & Michoacán & Queréndaro & E14-A14 & Fajr \\
\hline 81 & 49 & $100^{\circ} 45^{\prime}$ & $2 \bullet^{\circ} 50^{\prime}$ & 20 & Circular & NW-SE; N-S & Guanajuato & Calderón & F14-C55 & Fajr \\
\hline 82 & 49 & $100^{\circ} 43^{\prime}$ & $19^{\circ} 39^{\prime}$ & 10 & Circular & WNW-ESE; E-W & Michoacán & Mil Cumbres & E14-A24 & Good \\
\hline 83 & 49 & $100^{\circ} 39^{\prime}$ & $19^{\circ} 49^{\prime}$ & 10 & Semicircular & ENE-WSW & Michoacán & Ciudad Hidalgo & E14-A25 & Fajr \\
\hline 84 & 49 & $100^{\circ} 39^{\prime}$ & $19^{\circ} 45^{\prime}$ & 11 & Semicircular & ENE-WSW & Michoacán & Ciudad Hidalgo & E14-A24 & Poor \\
\hline 85 & 49 & $100^{\circ} 39^{\prime}$ & $19^{\circ} 49^{\prime}$ & 45 & Circular & $E-W$ & Michoacán & Mararatío & E14-A15 & Good \\
\hline 86 & 49 & $100^{\bullet} 37^{\prime}$ & $20^{\circ} 29^{\prime}$ & 9 & Semicircular & NW-SE & Guanajuato & Apaseo El Alto & $\mathrm{F} 14-\mathrm{C} 75$ & Fajr \\
\hline 87 & 49 & $100^{\bullet} 35^{\prime}$ & $20^{\circ} 50^{\prime}$ & 14 & Circular & ENE-WSW; NW-SE & Guanajuato & El Powero & $\mathrm{F} 14-\mathrm{C} 55$ & Fajr \\
\hline 88 & 42 & $100^{\circ} 28^{\prime}$ & $19^{\circ} 21^{\prime}$ & 5 & Circular & $\mathrm{E}-\mathrm{W}$ & Michoacán & La Florida & $\mathrm{E} 14-\mathrm{A} 35$ & Fajr \\
\hline 89 & 42 & $100^{\circ} 27^{\prime}$ & $19^{\circ} 24^{\prime}$ & 5 & Circular & E-W & Michoacán & Jungapeo & E14-A35 & Fajr \\
\hline 90 & 49 & $100^{\circ} 26^{\prime}$ & $20^{\circ} \cdot 4^{\prime}$ & $7 \times 10$ & Elliptical & E-W & Guanajuato & Puroaguita & F14-C85 & Poor \\
\hline 91 & 49 & $100^{\circ} 26^{\prime}$ & $19^{\circ} 31^{\prime}$ & 8 & Circular & $E-W$ & Michoacán & San Felipe de Alza & E14-A25 & Fair \\
\hline 92 & 42 & $100^{\circ} 23^{\prime}$ & $19^{\circ} 00^{\prime}$ & 16 & Circular & $\mathrm{N}-\mathrm{S}$ & Michoacán & Nanchiritla & E14-A 45, A46, A55, A56 & Fajr \\
\hline 93 & 49 & $100^{\circ} 21^{\prime}$ & $20^{\circ} 27^{\prime}$ & 5 & Semicircular & NE-SW; NW-SE & Querétaro & El Milagro & F14-C75 & Fajr \\
\hline 94 & 42 & $100^{\circ} 21^{\prime}$ & $19^{\circ} 30^{\prime}$ & $17 \times 25$ & Elliptical & $\mathrm{N}-\mathrm{S}$ & Michoacán & Zitácuaro & E14-A $34, \mathrm{~A} 35, \mathrm{~A} 24, \mathrm{~A} 25$ & Fair \\
\hline 95 & 42 & $100^{\circ} 20^{\prime}$ & $19^{\circ} 18^{\prime}$ & 2 & Circular & $\mathrm{N}-\mathrm{S}$ & México & Ixtapán del Oro & $\mathrm{E} 14-\mathrm{A} 35, \mathrm{~A} 36, \mathrm{~A} 45, \mathrm{~A} 46$ & Fajr \\
\hline 96 & 42 & $100^{\circ} 17^{\prime}$ & $19^{\circ} 28^{\prime}$ & 17 & Circular & $\mathrm{N}-\mathrm{S} ; \mathrm{E}-\mathrm{W}$ & Michoacán & Zitácuaro & E14-A35 & Good \\
\hline 97 & 42 & $100^{\circ} 13^{\prime}$ & $19^{\circ} 14^{\prime}$ & 15 & Circular & $\mathrm{NE}-\mathrm{SW}$ & México & Ixtapán del Oro & E14-A36, A46 & Fajr \\
\hline 98 & 41 & $100^{\circ} 12^{\prime}$ & $19^{\circ} 51^{\prime}$ & 8 & Semicircular & $E-W$ & Michoacán & Tlalpuj ahuillo & E14-A16 & Fajr \\
\hline 99 & 42 & $100^{\circ} 10^{\prime}$ & $18^{\circ} 51^{\prime}$ & $11 \times 13$ & Elliptical & ENE-WSW & México & Ixtapán de la Panocha & E14-A56 & Fajr \\
\hline 100 & 49 & $100^{\circ} 09^{\prime}$ & $20^{\circ} \cdot 8^{\prime}$ & 10 & Semicircular & $E-W$ & Querétaro & Amealco & F14-C86 & Good \\
\hline 101 & 41 & $100^{\circ} 08^{\prime}$ & $19^{\circ} 50^{\prime}$ & , & Semicircular & $\mathrm{E}-\mathrm{W}$ & Michoacán & Tlalpujahuillo & E14-A16 & Fajr \\
\hline 102 & 42 & $100^{\circ} \cdot 8^{\prime}$ & $19^{\circ} 3 \prime^{\prime}$ & 16 & Circular & E-W; N-S & México & Loma de Juarez & E14-A16, A17, A26, A27 & Good \\
\hline 103 & 49 & $100^{\circ} 07^{\prime}$ & $20^{\circ} 13^{\prime}$ & $32 \times 42$ & Elliptical & NE-SW; NW-SE & Querétaro & Colón & F14-C86 & Good \\
\hline 104 & 42 & $100^{\circ} 00^{\prime}$ & $19^{\circ} 28^{\prime}$ & 13 & Circular & $\mathrm{N}-\mathrm{S}$ & México & Villa Victoria & E14-A36, A37 & Good \\
\hline 105 & 41 & $100^{\circ} 00^{\prime}$ & $19^{\circ} 37^{\prime}$ & 8 & Circular & N-S; E-W & México & San Felipe del Progreso & E14-A26, A27, A16, A17 & Fajr \\
\hline 106 & 41 & $99^{\circ} 58^{\prime}$ & $19^{\circ} 55^{\prime}$ & 4 & Semicircular & $\mathrm{E}-\mathrm{W}$ & México & Temascalcingo & E14-A17 & Fajr \\
\hline 107 & 41 & $99^{\circ} 54^{\prime}$ & $20^{\circ} 25^{\prime}$ & 7 & Circular & $E-W$ & Querétaro & San Juan del Rio & $\mathrm{F} 14-\mathrm{C} 77$ & Poor \\
\hline 108 & 41 & $99^{\circ} 45^{\prime}$ & $20^{\circ} 26^{\prime}$ & 7 & Circular & N-S; E-W; NE-SW & Hidalgo & La Cruz & F14-C77 & Fajr \\
\hline 109 & 41 & $99^{\circ} 42^{\prime}$ & $19^{\circ} 33^{\prime}$ & 2 & Semicircular & $\mathrm{E}-\mathrm{W}$ & México & Sta. Cruz de Tepexpan & E14-A27 & Fajr \\
\hline $11 \bullet$ & 41 & $99^{\circ} 41^{\prime}$ & $19^{\circ} 39^{\prime}$ & 15 & Circular & E-W & México & Dolores Amarillas & E14-A27 & Fajr \\
\hline 111 & 42 & $99^{\circ} 41^{\prime}$ & $19^{\circ} 24^{\prime}$ & 16 & Circular & NE-SW; NW-SE & México & Alınoloya de Juárez & E14-A37 & Fair \\
\hline 112 & 42 & $99^{\circ} 38^{\prime}$ & $19^{\circ} 19^{\prime}$ & 32 & Circular & NW-SE & México & Toluca & E14-A38 & Fair \\
\hline 113 & 41 & $99^{\circ} 35^{\prime}$ & $20^{\circ} 18^{\prime}$ & 7 & Circular & $\mathrm{E}-\mathrm{W}$ & Hidalgo & Nopala & F14-C88 & Fajr \\
\hline 114 & 41 & $99^{\circ} 35^{\prime}$ & $19^{\circ} 30^{\prime}$ & , & Semicircular & $\mathrm{N}-\mathrm{S} ; \mathrm{E}-\mathrm{W}$ & México & Zanja Vieja & E14-A28 & Fajr \\
\hline 115 & 41 & $99^{\circ} 35^{\prime}$ & $19^{\circ} 25^{\prime}$ & 10 & Circular & $\mathrm{N}-\mathrm{S} ; \mathrm{E}-\mathrm{W}$ & México & Sta. Ana Jilotzingo & E14-A38 & Fajr \\
\hline 116 & 42 & $99^{\circ} 32^{\prime}$ & $19^{\circ} 14^{\prime}$ & 35 & Circular & ENE-WSW & México & Toluca + Tianguistanco & E14-A48 & Fair \\
\hline
\end{tabular}


Table 1 (continued)

\begin{tabular}{|c|c|c|c|c|c|c|c|c|c|c|}
\hline \multirow[t]{2}{*}{ Feature } & \multirow{2}{*}{$\begin{array}{l}\text { Landsat } \\
\text { image }\end{array}$} & \multicolumn{2}{|l|}{ Coordinates } & \multirow{2}{*}{$\begin{array}{l}\text { Diameter } \\
(\mathrm{km})\end{array}$} & \multirow[t]{2}{*}{ Morphology } & \multirow[t]{2}{*}{ Lineaments } & \multirow{2}{*}{$\begin{array}{l}\text { Political } \\
\text { State }\end{array}$} & \multirow[t]{2}{*}{ Closest village } & \multirow{2}{*}{$\begin{array}{l}\text { INEGI } \\
\text { map no. }\end{array}$} & \multirow{2}{*}{$\begin{array}{l}\text { Feature } \\
\text { reliability }^{b}\end{array}$} \\
\hline & & Long. (W) & Lat. (N) & & & & & & & \\
\hline 117 & 41 & $99^{\circ} 31^{\prime}$ & $20^{\circ} 21^{\prime}$ & $8 \times 10$ & Elliptical & - & Hidalgo & Huichapan & F14-C88 & Good \\
\hline 118 & 41 & $99^{\circ} 31^{\prime}$ & $19^{\circ} 46^{\prime}$ & 4 & Circular & N-S; E-W & México & San Fco. de la Tablas & E14-A18 & Fajr \\
\hline 119 & 41 & $99^{\circ} 30^{\prime}$ & $19^{\circ} 52^{\prime}$ & 3 & Semicircular & $\mathrm{N}-\mathrm{S} ; \mathrm{E}-\mathrm{W}$ & México & Barajas & E14-A18 & Fajr \\
\hline 12 & 41 & $99^{\circ} 30^{\prime}$ & $19^{\bullet} 35^{\prime}$ & 12 & Circular & N-S; E-W; NW-SE & México & Jiquilpingo & E14-A28 & Good \\
\hline 121 & 41 & $99^{\circ} 29^{\prime}$ & $20^{\circ} \cdot 3^{\prime}$ & 11 & Circular & NE-SW; NW-SE & México & San Agusún Buenavista & F14-C88 & Fajr \\
\hline 122 & 41 & $99^{\circ} 26^{\prime}$ & $20^{\bullet} 11^{\prime}$ & $4 \times 6$ & Elliptical & $E-W$ & Hidalgo & Tepetitlán & F14-C88 & Good \\
\hline 123 & 41 & $99^{\circ} 24^{\prime}$ & $20^{\circ} \cdot 3^{\prime}$ & 3 & Circular & NE-SW & Hidalgo & Tula de Allende & F14-C88 & Fajr \\
\hline 124 & 41 & $99^{\circ} 23^{\prime}$ & $19^{\circ} 28^{\prime}$ & 8 & Elliptical & $\mathrm{N}-\mathrm{S} ; \mathrm{E}-\mathrm{W}$ & México & Villa Alpina & E14-A38 & Fajr \\
\hline 125 & 42 & $99^{\circ} 20^{\prime}$ & $18^{\bullet} 59^{\prime}$ & 7 & Circular & NE-SW; N-S & México & Ocuilán & E14-A58, A59 & Fajr \\
\hline 126 & 42 & $99^{\circ} 17^{\prime}$ & $18^{\bullet} 53^{\prime}$ & 18 & Circular & $\mathrm{N}-\mathrm{S}$ & Morelos & Cuemavaca & E14-A59 & Fajr \\
\hline 127 & 42 & $99^{\bullet} 17^{\prime}$ & $19^{\circ} 22^{\prime}$ & 15 & Circular & NNW-SSE; E-W & D.F. & San Miguel Ajusco & E14-A39 & Fajr \\
\hline 128 & 41 & $99^{\circ} 15^{\prime}$ & $20^{\bullet} 28^{\prime}$ & 3 & & Semielliptical & $N-S ; E-W$ & Hidalgo & Ixmiquilpan & F14-C79 \\
\hline \multicolumn{11}{|l|}{ Fajr } \\
\hline 129 & 41 & $99^{\circ} 13^{\prime}$ & $20^{\circ} 17^{\prime}$ & $17 \times 18$ & Elliptical & ENE-WSW; NW-SE & Hidalgo & Progreso & F14-C89 & Good \\
\hline 130 & 41 & $99^{\circ} 11^{\prime}$ & $19^{\circ} 45^{\prime}$ & 4 & Semicircular & N-S; E-W & México & El Sitio & E14-B11 & Poor \\
\hline 131 & 41 & $99^{\circ} 10^{\prime}$ & $20^{\bullet} 19^{\prime}$ & 5 & Semicircular & E-W; ENE-WSW & Hidalgo & Progreso & F14-C89 & Fajr \\
\hline 132 & 42 & $99^{\circ} 99^{\prime}$ & $19^{\circ} 15^{\prime}$ & 5 & Circular & $E-W$ & D.F. & Topilejo & E14-A48, A38, A49, A39 & Fajr \\
\hline 133 & 42 & $99^{\circ} 03^{\prime}$ & $19^{\bullet} 18^{\prime}$ & $10 \times 15$ & Elliptical & E-W & D.F. & Xochimilco & E14-A49 & Fajr \\
\hline 134 & 34 & $99^{\circ} 03^{\prime}$ & $19^{\circ} 48^{\prime}$ & 9 & Circular & - & México & San Pedro dela Laguna & E14-A19 & Fajr \\
\hline 135 & 41 & $99^{\circ} 00^{\prime}$ & $20^{\circ} 02^{\prime}$ & 10 & Semicircular & NNW-SSE; NE-SW & Hidalgo & Ajacuba & - & Fajr \\
\hline 136 & 34 & $98^{\circ} 57^{\prime}$ & $19^{\circ} 52^{\prime}$ & 4 & Circular & $\mathrm{N}-\mathrm{S}$ & México & Santa Lucia & E14-B11 & Fajr \\
\hline 137 & 35 & $98^{\bullet} 57^{\prime}$ & $19^{\circ} 17^{\prime}$ & 3 & Circular & NE-SW & D.F & Sta. María Nativitas & E14-B31 & Good \\
\hline 138 & 35 & $98^{\bullet} 57^{\prime}$ & $19^{\circ} 26^{\prime}$ & 14 & Circular & NE-SW & D.F. & Sta. María Nativitas & E14-B31 & Fajr \\
\hline 139 & 34 & $98^{\bullet} 53^{\prime}$ & $19^{\circ} 47^{\prime}$ & 6 & Circular & NW-SE & México & Santa Lucía & E14-B11 & Poor \\
\hline 140 & 41 & $98^{\circ} 53^{\prime}$ & $20^{\bullet} 25^{\prime}$ & 8 & Circular & NW-SE; E-W & Hidalgo & Santiago de Anaya & E14-A81 & Good \\
\hline 141 & 35 & $98^{\circ} 52^{\prime}$ & $19^{\circ} 4^{\prime}$ & 8 & Circular & $E-W$ & México & Juenitepec de Mariano & E14-B41 & Good \\
\hline 142 & 41 & $98^{\circ} 50^{\prime}$ & $20^{\bullet} 24^{\prime}$ & 8 & Circular & NW-SE; E-W & Hidalgo & Santiago de Anaya & F14-A81 & Good \\
\hline 143 & 41 & $98^{\circ} 50^{\prime}$ & $20^{\bullet} 23^{\prime}$ & 7 & Circular & NW-SE; E-W & Hidalgo & Santiago de Anaya & F14-A81 & Fajr \\
\hline 144 & 35 & $98^{\circ} 49^{\prime}$ & $19^{\circ} 4^{\prime}$ & 6 & Circular & $E-W$ & México & Nepantla & E14-B41 & Good \\
\hline 145 & 41 & $98^{\circ} 49^{\prime}$ & $19^{\circ} 45^{\prime}$ & 2.5 & Circular & NNW-SSW; E-W & México & Teacalco & E14-B11 & Fajr \\
\hline 146 & 34 & $98^{\circ} 49^{\prime}$ & $19^{\bullet} 57^{\prime}$ & 10 & Circular & - & Hidalgo & Zapatlán de Juárez & E14-B11 & Poor \\
\hline 147 & 34 & $98^{\circ} 48^{\prime}$ & $19^{\circ} 50^{\prime}$ & 6 & Semicircular & WNW-ESE & México & Teopancala & E14-B11 & Fajr \\
\hline 148 & 34 & $98^{\circ} 46^{\prime}$ & $19^{\circ} 41^{\prime}$ & 12 & Circular & NE-SW & México & Teotihuacán & E14-B11 & Poor \\
\hline 149 & 35 & $98^{\circ} 46^{\prime}$ & $19^{\circ} 01^{\prime}$ & 9 & Circular & $E-W$ & México & Tepetlixpa & E14-B41 & Fajr \\
\hline 15 & 34 & $98^{\circ} 46^{\prime}$ & $20^{\circ} \cdot 3^{\prime}$ & 11 & Circular & - & Hidalgo & Huiquilpan & F14-D81 & Poor \\
\hline 151 & 34 & $98^{\circ} 40^{\prime}$ & $20^{\circ} \cdot 2^{\prime}$ & $13 \times 2$ & Elliptical & - & Hidalgo & Epazoyucán & - & Fajr \\
\hline 152 & 34 & $98^{\bullet} 36^{\prime}$ & $20^{\bullet} 15^{\prime}$ & 14 & Circular & NW-SE & Hidalgo & Atotonilco el Grande & - & Fajr \\
\hline 153 & 34 & $98^{\circ} 34^{\prime}$ & $20^{\circ} 00^{\prime}$ & 5 & Circular & - & Hidalgo & Zempoala & - & Fajr \\
\hline 154 & 34 & $98^{\bullet} 34^{\prime}$ & $20^{\circ} 01^{\prime}$ & 5 & Circular & - & Hidalgo & Epazoyucán & - & Fajr \\
\hline
\end{tabular}


Table 1 (continued)

\begin{tabular}{|c|c|c|c|c|c|c|c|c|c|c|}
\hline \multirow[t]{2}{*}{ Feature } & \multirow{2}{*}{$\begin{array}{l}\text { Landsat } \\
\text { image }\end{array}$} & \multicolumn{2}{|l|}{ Coordinates } & \multirow{2}{*}{$\begin{array}{l}\text { Diameter } \\
(\mathrm{km})\end{array}$} & \multirow[t]{2}{*}{ Morphology } & \multirow[t]{2}{*}{ Lineaments } & \multirow{2}{*}{$\begin{array}{l}\text { Political } \\
\text { State }\end{array}$} & \multirow[t]{2}{*}{ Closest village } & \multirow{2}{*}{$\begin{array}{l}\text { INEGI } \\
\text { map no" }\end{array}$} & \multirow{2}{*}{$\begin{array}{l}\text { Feature } \\
\text { reliability }^{b}\end{array}$} \\
\hline & & Long. (W) & Lat. (N) & & & & & & & \\
\hline 155 & 34 & $98^{\bullet} 26^{\prime}$ & $20^{\circ} \cdot 6^{\prime}$ & 5 & Circular & NE-SW & Hidalgo & Acatlán & - & Poor \\
\hline 156 & 34 & $98^{\bullet} 25^{\prime}$ & $20^{\bullet} 56^{\prime}$ & 6 & Semicircular & E-W & Hidalgo & Singuilucan & - & Fajr \\
\hline 157 & 34 & $98^{\circ} 20^{\prime}$ & $19^{\circ} \cdot 4^{\prime}$ & $18 \times 22$ & Elliptical & NE-SW & Hidalgo & Tulancingo & - & Fajr \\
\hline 158 & 34 & $98^{\bullet} 12^{\prime}$ & $18^{\bullet} 58^{\prime}$ & $10 \times 12$ & Elliptical & E-W & Puebla & Puebla & - & Fajr \\
\hline 159 & 34 & $98^{\circ} 10^{\prime}$ & $19^{\bullet} 34^{\prime}$ & 16 & Circular & - & Tlaxcala & Tlaxco & - & Poor \\
\hline 16 & 34 & $98^{\circ} 09^{\prime}$ & $19^{\circ} 58^{\prime}$ & $5 \times 9$ & Elliptical & - & Puebla & Acoculco & - & Poor \\
\hline 161 & 34 & $98^{\circ} 03^{\prime}$ & $19^{\bullet} 33^{\prime}$ & 20 & Circular & NW-SE; NE-SW & Tlaxcala & Atotonilco & - & Fajr \\
\hline 162 & 34 & $97^{\circ} 59^{\prime}$ & $19^{\circ} 31^{\prime}$ & 3 & Circular & ENE-WSW & Tlaxcala & Terrenate & - & Fajr \\
\hline 163 & 35 & $97^{\circ} 45^{\prime}$ & $19^{\circ} 19^{\prime}$ & $12 \times 15$ & Elliptical & E-W & Tlaxcala & Xicoténcatl & - & Fajr \\
\hline 164 & 29 & $97^{\circ} 44^{\prime}$ & $19^{\circ} 0 \mathbf{o}^{\prime}$ & 5 & Circular & $\mathrm{N}-\mathrm{S}$ & Puebla & Acatcingo & - & Poor \\
\hline 165 & 35 & $97^{\circ} 40^{\prime}$ & $19^{\circ} 13^{\prime}$ & 12 & Circular & - & Puebla & Rafael Lara Grajales & - & Fajr \\
\hline 166 & 35 & $97^{\bullet} 35^{\prime}$ & $19^{\bullet} 17^{\prime}$ & 15 & Circular & - & Puebla & Oriental & - & Fajr \\
\hline 167 & 29 & $97^{\bullet} 34^{\prime}$ & $19^{\bullet} 33^{\prime}$ & 5 & Circular & $\mathrm{N}-\mathrm{S}$ & Puebla & Rafael Lara Grajales & - & Good \\
\hline 168 & 29 & $97^{\bullet} 33^{\prime}$ & $19^{\circ} 08^{\prime}$ & 9 & Circular & $\mathrm{N}-\mathrm{S}$ & Puebla & San Salvador el Seco & - & Good \\
\hline 169 & 28 & $97^{\bullet} 28^{\prime}$ & $19^{\circ} 23^{\prime}$ & 4 & Circular & $\mathrm{N}-\mathrm{S}$ & Puebla & Cerro Pinto & - & Good \\
\hline 17 & 28 & $97^{\circ} 26^{\prime}$ & $19^{\circ} 4 \mathbf{\prime}^{\prime}$ & 17 & Circular & WNW-ESE & Puebla & Tezuitlán & - & Good \\
\hline 171 & 29 & $97^{\bullet} 26^{\prime}$ & $19^{\circ} 15^{\prime}$ & $8 \times 10$ & Elliptical & $\mathrm{N}-\mathrm{S}$ & Puebla & Emilio Portes Gil & - & Good \\
\hline 172 & 28 & $97^{\bullet} 23^{\prime}$ & $19^{\circ} 40^{\prime}$ & 50 & Circular & - & Puebla & Tezuitlán & - & Good \\
\hline 173 & 29 & $97^{\bullet} 23^{\prime}$ & $19^{\bullet} 14^{\prime}$ & $7 \times 9$ & Elliptical & $\mathrm{N}-\mathrm{S}$ & Puebla & Zacatepec & - & Good \\
\hline 174 & 29 & $97^{\bullet} 18^{\prime}$ & $19^{\circ} 27^{\prime}$ & 8 & Circular & NE-SW & Veracruz & San Antonio Limón & - & Good \\
\hline 175 & 29 & $97^{\bullet} 15^{\prime}$ & $19^{\circ} 15^{\prime}$ & 3 & Circular & $\mathrm{N}-\mathrm{S}$ & Veracruz & Cerro de las Cumbres & - & Good \\
\hline 176 & 29 & $97^{\circ} 10^{\prime}$ & $19^{\circ} 20^{\prime}$ & $9 \times 12$ & Elliptical & - & Veracruz & Perote & - & Poor \\
\hline 177 & 29 & $97^{\circ} 99^{\prime}$ & $19^{\circ} 9^{\prime}$ & $5 \times 7$ & Elliptical & NW-SE & Veracruz & Perote & - & Poor \\
\hline 178 & 29 & $97^{\circ} 18^{\prime}$ & $19^{\circ} 19^{\prime}$ & 6 & Semicircular & NW-SE; NE-SW & Puebla & Chichiquitán & - & Good \\
\hline 179 & 29 & $97^{\bullet} 08^{\prime}$ & $19^{\circ} 04^{\prime}$ & $16 \times 25$ & Elliptical & $\begin{array}{l}\text { NW-SE; NE-SW; E- } \\
\text { W }\end{array}$ & Veracruz & Coscomatepec & - & Good \\
\hline 18 & 29 & $97^{\circ} 06^{\prime}$ & $19^{\bullet} 21^{\prime}$ & 5 & Semicircular & NW-SE & Veracruz & Ayahualulco & - & Good \\
\hline 181 & 29 & $97^{\circ} 05^{\prime}$ & $19^{\bullet} 33^{\prime}$ & 20 & Circular & WNW-ESE & Veracruz & Perote & - & Good \\
\hline 182 & 29 & $97^{\bullet} 05^{\prime}$ & $19^{\circ} 28^{\prime}$ & 7 & Elliptical & NW-SE & Veracruz & Coatitlán & - & Good \\
\hline 183 & 29 & $97^{\bullet} 93^{\prime}$ & $19^{\bullet} 17^{\prime}$ & 10 & Circular & NW-SE; NE-SW & Puebla & Chichiquitán & - & Good \\
\hline 184 & 29 & $97^{\circ} 92^{\prime}$ & $19^{\circ} 26^{\prime}$ & 3 & Circular & NW-SE & Veracruz & Coatitlán & - & Good \\
\hline 185 & 29 & $97^{\bullet} 56^{\prime}$ & $19^{\circ} 24^{\prime}$ & 8 & Circular & NW-SE & Veracruz & Coatitlán & - & Good \\
\hline 186 & 29 & $96^{\circ} 52^{\prime}$ & $19^{\bullet} 33^{\prime}$ & $4 \times 5$ & Elliptical & E-W & Veracruz & Jalapa Enríquez & - & Fair \\
\hline 187 & 29 & $96^{\circ} 51^{\prime}$ & $19^{\circ} 28^{\prime}$ & 9 & Circular & NW-SE & Veracruz & Jalapa Enríquez & - & Poor \\
\hline 188 & 29 & $96^{\circ} 48^{\prime}$ & $19^{\circ} 29^{\prime}$ & 9 & Circular & WNW-ESE & Veracruz & Jalapa Enríquez & - & Poor \\
\hline 189 & 29 & $96^{\circ} 41^{\prime}$ & $19^{\circ} 25^{\prime}$ & 17 & Circular & WNW-ESE & Veracruz & Cosautlán de Carvajal & - & Poor \\
\hline 190 & 29 & $96^{\circ} 40^{\prime}$ & $19^{\circ} 23^{\prime}$ & 45 & Circular & WNW-ESE & Veracruz & Actopán & - & Fajr \\
\hline 191 & 29 & $96^{\bullet} 38^{\prime}$ & $19^{\circ} 24^{\prime}$ & 4 & Circular & E-W & Veracruz & Idolos & - & Good \\
\hline
\end{tabular}

a - , INEGI 1:50,000 map not available.

b Feature reliability: 'Good' means that the feature is clearly visible (e.g. Fig. 2a); 'Poor' is reserved for dubious features (e.g. Fig. 2b). 
al., 1991; see Fig. 4 for locations of these calderas). The $\bullet$ ther known calderas are alsø clearly visible $\bullet$ Landsat imagery. A comprehensive evaluation of pøtential geothermal resøurces and volcanic hazards within the Trans-Mexican Vølcanic Belt could benefit frøm our apprøach.

\section{Acknowledgements}

This work was partly supported by DGAPA project N-106199. The first author (F.A.) thanks the Universidad Complutense-UNAM bilateral Program for a partial support, enabling him to visit the UNAM. Copies of Landsat spaciømaps were donated by J.F. Allan t॰ one of us (S.P.V.). Computer drawing of the TMVB sketch was carried out by Jøsé Møjarrø. We are als@ grateful t• Margaret T. Mangan and W.A. Duffield for constructive comments on an earlier version of this paper.

\section{References}

Allan, J.F., Nelson, S.A. Lubr, J.F., Cannichael, I.S.E., Wopat, M., Wallace, P.J., 1991. Pliocene-Holocene rifting and associated volcanism in southwest Mexico: an exotic terrane in the making. In: Dauphin, J.P., Simoneit, B.R.T. (Eds.), The Gulf and Peninsular Province of the Californias. Am. Assoc. Petrol. Geol. Memoir 47, pp. 425-445.

Anguita, F., Venna, S.P., García-Cacho, L., Milán, M., Samaniego, D., 1991. Mazahua: una nueva caldera en el Cinturón Volcánico Mexicano. Geofís. Int. 31, 135-148.

Campos-Enríquez, J.O., Rodríguez, M., Delgado-Rodríguez,O., Milán, M., 1999. A conwibution to the tectonics of the Northern portion of the central sector of the Trans-Mexican Volcanic Belt. In: Cenozoic Volcanism and Tectonics of Mexico. Geol. Soc. Am. Spec. Paper 334 (in press).

Demant, A., 1978. Características del eje neovolcánico transmexicano y sus problemas de interpretación. UNAM Inst. Geol. Rev. 2, 172-187.

Ferrari, L., Garduño, V.H., Pasquarè, G., Tibaldi, A., 1991. Geology of Los Azufres caldera, Mexico, and its relationships with regional tectonics. J. Volcanol. Geotherm. Res. 47, 129-148.
Jolmson, C.A., Harrison, C.G.A., 1990. Neotectonics in central Mexico. Phys. Earth Planet. Int. 64, 187-210.

Lugo, J., Ortiz, MA., Palacio, J.L., Bocco, G., 1985. Las zonas más activas en el Cinturón Volcánico Mexicano (entre Michoacán y Tlaxcala). Geofís. Int. 24, 83-96.

Lubr, J.F., Nelson, S.A., Allan, J.F., Carmichael, I.S.E., 1985. Active rifting in Southwestern Mexico: manifestations of an incipient eastward spreading-ridge jump. Geology 13, 54-57.

Moore, G., Marone, C., Carmichael, I.S.E., Renne, P., 1994. Basalic volcanism and extension near the intersection of the Sierra Madre volcanic province and the Mexican Volcanic Belt. Geol. Soc. Am. Bull. 106, 383-394.

Negendank, J.F.W., Emmermann, R., Krawczyk, R., Mooser, F., Tobschall, H., Werle, D., 1985. Geological and geochemical investigations on the eastern Trans-Mexican Volcanic Belt. In: Venna, S.P. (Ed.), Spec. Vol. on the Mexican Volcanic Belt, part 2. Geofís. Int. 24, 477-575.

Nelson, S.A., Cannichael, I.S.E., 1984. Pleistocene to Recent alkalic volcanism in the region of Sanganguey volcano, Nayarit, Mexico. Con rib. Mineral. Petrol. 85, 321-335.

Nelson, SA., Sánchez-Rubio, G., 1986. Trans-Mexican Volcanic Belt field guide. Volcanol. Div. Geol. Assoc. Canada-UNAM, 79-108.

Pasquarè, G., Ferrari, L., Perazzoli, V., Tiberi, M., Turchetii, F., 1987. Morphological and structural analysis of the central sector of the Trans-Mexican Volcanic Belt. Geofís. Int. 26, $177-193$.

Pradal, E., Robin, C., 1985. Découverte d'une caldera majeure associée au champ géothennique de Los Azufres (Méxique). C.R. Acad. Sci. Paris S. II 14, 31-316.

Pérez-Reynoso, J., 1978. Geología y perrografía dela caldera de Los Humeros. Geomimet ( $3^{\mathrm{a}}$ Época) 91, 97-106.

Ramírez-Herrera, M.T., 1996. Morphological evidence for neotectonic activity and seismic hazard in the Acambay graben, Mexican Volcanic Belt. In: Slaymaker, O. (Ed.), Geomorphic Hazards. Wiley, Chichester, UK, pp. 29-41.

Robin, C., 1982. Mexico. In: Thorpe, R.S. (Ed.), Andesites. Wiley, Chichester, UK, pp. 137-147.

Suter, M., Quintero-Legorreta, O., López-Martínez, M., AguirreDíaz, G., Farrar, E., 1995. The Acambay graben: activein raarc extension in the Trans-Mexican Volcanic Belt, Mexico. Tectonics 14, 1245-1262.

Venna, S.P., 1987. Mexican Volcanic Belt: present state of knowledge and unsolved problems. Geofís. Int. 26, 309-34t.

Wallawender, M.J., Hanan, B.B., 1992. Geological excursions in Southern California and Mexico. Guidebook 1991 Annual Meeting of the Geological Society of America, pp. 224-243. 